\title{
"You have to get wet to learn how to swim" applied to bridging the gap between research into personnel scheduling and its implementation in practice
}

\author{
Sanja Petrovic $^{1}$ (D)
}

Published online: 8 July 2017

(C) The Author(s) 2017. This article is an open access publication

\begin{abstract}
Personnel scheduling problems have attracted research interests for several decades. They have been considerably changed over time, accommodating a variety of constraints related to legal and organisation requirements, part-time staff, flexible hours of staff, staff preferences, etc. This led to a myriad of approaches developed for solving personnel scheduling problems including optimisation, meta-heuristics, artificial intelligence, decisionsupport, and also hybrids of these approaches. However, this still does not imply that this research has a large impact on practice and that state-of-the art models and algorithms are widely in use in organisations. One can find a reasonably large number of software packages that aim to assist in personnel scheduling. A classification of this software based on its purpose will be proposed, accompanied with a discussion about the level of support that this software offers to schedulers. A general conclusion is that the available software, with some exceptions, does not benefit from the wealth of developed models and methods. The remaining of the paper will provide insights into some characteristics of real-world scheduling problems that, in the author's opinion, have not been given a due attention in the personnel scheduling research community yet and which could contribute to the enhancement of the implementation of research results in practice. Concluding remarks are that in order to bridge the gap that still exists between research into personnel scheduling and practice, we need to engage more with schedulers in practice and also with software developers; one may say we need to get wet if we want to learn how to swim.
\end{abstract}

Keywords Personnel scheduling $\cdot$ Software for personnel scheduling $\cdot$ Personnel scheduling research directions

Sanja Petrovic

sanja.petrovic@nottingham.ac.uk

1 Nottingham University Business School, Jubilee Campus, Wollaton Road, Nottingham,

NG8 1BB, UK 


\section{Introduction}

Personnel scheduling can be defined as the process of assignment of personnel to shifts to cover the demand for resources that varies over time (Ernst et al. 2004b). The personnel scheduling problems exist almost everywhere, in service industry such as health care sector (nurse rostering, scheduling of surgeons, etc.), transport and logistic sector (crew rostering and crew scheduling in airlines, railways, bus companies, mass transit companies, etc.), civic services (libraries, post-offices, universities, etc.) call-centres, manufacturing, venue management.

Personnel scheduling has attracted research interests since 1950s (Edie 1954). Personnel scheduling problems have been considerably changed since then, accommodating a variety of constraints related to legal and organisation requirements, part-time staff, flexible hours of staff, staff preferences, etc. This led to a myriad of approaches developed for solving personnel scheduling problems, which can be classified in the following groups: optimisation, meta-heuristics, artificial intelligence, decision-support, and also hybrids of these approaches. There is a large volume of personnel scheduling literature, which consequently led to a number of survey papers. They offer different classification of personnel scheduling problems and provide comprehensive collections of the relevant literature (Burke et al. 2004; Ernst et al. 2004b; Van den Bergh et al. 2013). An annotated bibliography of more than 700 papers has been collated by Ernst et al. (2004a). The papers were organised by the type of scheduling problem they addressed and by the application area. There are numerous benefits of generating high quality personnel schedules which include: reduced cost of human resources (in many organizations the cost of personnel forms the major part of the direct cost), better provision of service (for example, satisfying adequately the demand for service with skilled personnel, or providing consistency in personnel offering service, where it is relevant), increase in the job satisfaction of personnel, increase of the well-being of personnel, retention of the personnel, increase in the perception of the fairness of a schedule. Ultimately, these can assist in the recruitment of new personnel. Therefore, the impact of research into personnel scheduling on practice has high potentials. However, as Jan van de Snepscheut, California Institute of Technology, noted "In theory, there is no difference between theory and practice. But, in practice, there is."

Due to the advances in technology it becomes more and more common that managers use some software packages to assist them to greater or smaller extent in generating personnel schedules. Such software packages usually include a variety of features to enable managers to easily create and employees to easily access the schedule. Common features include import/export of data describing employees, access to the software through mobile devices, access to the software through cloud servers, which means that the software can be accessed from any computer with an internet connection, posting schedules online so that employees can view them on their computers or mobile devices, sending text and/or e-mails to employees reminding them of their shifts, etc. This gives a great opportunity to employ computer science and software development knowledge to advance personnel scheduling. Some additional nice features include the ability to click and drag an employee's name into a schedule, and synchronisation of the employee schedule with his/her calendar applications. However, the main question of interest here is how powerful the scheduling algorithms that these software packages employ are? Do they represent the state-of-the-art in the personnel scheduling research community?

It is recognised that Operational Research techniques can produce high quality rosters, but that they have not been fully exploited and implemented in healthcare (Utley 2016). There is hardly any research study about the implementation of developed methods for personnel 
scheduling in practice. To the best of our knowledge the only paper was published by Kellogg and Walczak (2007) and it was focused on nurse scheduling. The authors thoroughly investigated 50 models and methods published in the literature in the period 1985-2005 and their implementation in practice. The authors gathered required information from published papers and also by contacting the authors via e-mails. They reported that 15 models $(30 \%)$ were implemented, while six of them were still in use in 2007 (the remaining ones were not in use or their usage was not known). This paper does not aim to repeat this endeavour and investigate if there have been any changes in the recent time period. It would be difficult to involve software vendors in the study because generally they do not share the details of the methods they employ in their software. Instead, the paper will propose a classification of the software packages for personnel scheduling based on desk research of the current software market, and discuss features they offer. The names of investigated software packages will be omitted as the purpose of this discussion is to report on the common characteristics that "sell" the software for personnel scheduling and not to advertise any particular software.

It is viewed that the airline crew-scheduling problems are different to standard personnel scheduling problems, mostly because of their structure, i.e. they usually consist of two sub-problems: the planning or pairing problem, which involves the construction of feasible sequences of duty and rest periods, and the rostering problem in which the pairings are assigned to individual crew members (Gopalakrishnan and Johnson 2005). They also have some specific constraints, such as the start and finish times of each task are pre-determined, the crew physically moves between locations including locations across countries with different time zones, staff face substantial irregularity in work-rest patterns, etc. The employees are the largest single cost to the airlines and therefore many of the companies use specialised software packages for crew scheduling. The use of these software packages lead to considerable savings (Gopalakrishnan and Johnson 2005; Butchers et al. 2001). Due to the very specific characteristics of this type of personnel scheduling problem, it will not be included in our discussion in depth. However, some insights into the possible reasons of successful implementation of research in the airline crew-scheduling reported in the literature will be provided.

The discussion about software for personnel scheduling will be followed by insights into some characteristics of real-world scheduling problems, which, in the author's opinion, have still to be given a due attention in the personnel scheduling research community. There is still a gap between research into personnel scheduling and practice. If we aim to enhance the implementation of research results in practice and bridge the existing gap, we need to engage more with schedulers in practice and also with software developers; one may say we need to get wet if we want to swim.

The structure of the paper is as follows. The definition of personnel scheduling problem is given in Sect. 2 followed by a brief overview of methods employed in personnel scheduling. A discussion about software for personnel scheduling and its classification is provided in Sect. 3. Insights into some characteristics of real-world scheduling problems that would need to be better addressed in the methods for personnel scheduling are presented in Sect. 4. The paper completes with some concluding remarks.

\section{Personnel scheduling-problem definition and methods}

Personnel scheduling (also referred to as employee timetabling and rostering) can be stated as a mathematical optimisation problem in which a work timetable for staff is constructed to satisfy the demand for services or goods (Ernst et al. 2004a, b). In organisations in which 
demand varies considerably over time, personnel scheduling is difficult problem to solve. The personnel can be homogeneous i.e. can consist of one type of full-time employees, or mixed when there are several types of full-time and part-time employees. The construction of a schedule is subject to given rules/constraints. These are usually divided into two categories: "hard" and "soft". Hard constraints have to be satisfied in a schedule; for example, a required number of personnel with required skills must be scheduled in each shift. Opposite them, the satisfaction of soft constraints is desirable, but not essential. For example, working night shifts in a block or working both weekend days are often soft constraints. If it is not possible to find a solution that satisfies all of the soft constraints, which happens often in real-world personnel scheduling, then the number of violations of soft constraints or the extent of the violations serve as indicators of the schedule performance. The constraints imposed on scheduling generally make the set of all possible solutions (i.e. the search space of the problem) very large. In practice, personnel scheduling problems can be very hard to solve, and their manual constructing is time-consuming process which requires much effort.

Workforce planning (also referred to as the staffing problem) deals with strategic decisions related to the optimal size and mix of workforce. Also, for the given demand usually expressed by statistical data, shifts required to cover the demand are determined, together with a personnel structure required to meet the demand. The latter can be described by the contract type, knowledge required, skills, etc. These serve as input to a scheduling problem. In general, planning precedes personnel scheduling, i.e. in the scheduling problem it is assumed that the staffing, together with shifts and personnel structure are given.

Smet et al. (2014) introduced a general model for personnel scheduling, which was applicable to a variety of personnel scheduling problems. The following components of a schedule were identified which are enriched here with additional observations and examples:

- Rostering period varies among different sectors, countries and time of the year. It can be a day, fortnights, 4 weeks, etc. Also, some periods such as Christmas and summer holidays may require a different treatment than the rest of the year due to the fewer available personnel and consequently the scheduling period can be shorter, for example, 2 weeks instead of standard 4 weeks.

- Skill types depend on the application. In some applications, skill types are organised hierarchically and often personnel on higher ranks are allowed to carry out tasks of lower ranks but not vice versa. However, personnel on high ranks incur more costs. In some applications due to the training purpose employee with less training have to be scheduled with more senior employees; for example, students have to be grouped together in the same shift with physicians or nurses. Additional common characteristic of personnel scheduling models and algorithms is that they consider the skills of personnel to be fixed and serve as input.

- Shift types refer to daily assignment units. They are defined by a specific start and end time. In most problems, a shift type requires rest period before and after the shift. There are also breaks during shifts, which have to be taken into account when calculating the net job time.

- Constraint/rule set apply restrictions to the roster of an employee. They are also referred to as horizontal constraints or time related constraints. There are three types of constraints: (1) to count the number of specific roster items such as hours worked, shift type worked, weekends worked, etc; (2) to restrict consecutive occurrences of specific roster items, e.g. shift type worked, days worked, non-working days, etc., and (3) to control the succession of two series, i.e. to ensure that one occurrence of specific roster items is followed by 
another, e.g. number of specific shift type worked should be followed by a given number of non-working days.

- Employees described by employee skill type, contract hours, availability, etc. For example, employee can be characterised by requests for absence and assignments, salary, etc.

- Schedule a set of assignments where an assignment consists of four elements: the employee, the date, the shift type and the required skill type.

- Schedule constraints the number of employees needed for each shift and required skill types.

- Teams teams of employees with complementary skills have to work together. Employees who should not work together can be also defined.

- Training controls the ratio between employees with different levels of experience.

Typical objectives considered in the personnel scheduling are as follows:

- To minimise the total cost which is the sum of the costs of individual schedules; this may be a combination of salary, travel and accommodation costs.

- To maximise the satisfaction of constraints which include employees' satisfaction: The objective function usually counts the number of constraint violations in the schedule or the extent to which they are violated. Violations can be weighted according to the perceived relative importance of their corresponding constraint type.

- To maximise the service quality in service organisations e.g. call-centres. It can be measured by the service level (the percentage of customers served within a predefined time interval), average/maximum waiting time, average/maximum queue length, abandonment rate (the percentage of customers who abandon queue prior to the service), blocking rate (the percentage of customers who attempt but are not able to join the queue), personnel utilisation (the percentage of busy time over total time of employee), etc. (Castillo et al. 2009).

Self-scheduling (often referred to as self-rostering) aims at giving more control over assigned shifts to staff. It was first introduced in the 1960s at a London hospital (Bailyn 2007). However, self-scheduling did not catch on until the 1980s. It is now being used in many institutions, especially health care institutions, and serves well as a staff retention tool by satisfying the individual preferences of staff, increasing their morale and offering flexible scheduling options. It has been implemented only in wards of small size. The benefits of self-rostering are well documented (Hung 2002). Self-rostering imposes requirements that must be met by all personnel signing up, such as the requirement for a number of working weekends over the rostering period; it must be signed up evenly and some sort of signed up order has to be considered, for example first come-first get. The rostering has to satisfy the coverage requirements. In order for the self-rostering to be successful, staff members have to consider not only their own needs and preferences, but also the needs of the institution. Accordingly, self-rostering is viable only if it includes specific rules designed to ensure fairness. It was reported in the literature that rosters produced using self-rostering within e-rostering require fewer post-approval changes and when changes are made they break fewer rostering rules (Drake 2014). However, although self-rostering has been found to be successful, it also faces difficulties. Silvestro and Silvestro (2000) identify the disadvantages of self-rostering: the process can easily lead to overstaffing or understaffing; it is not suitable for complex problems; the overhead of training all staff to understand rostering problem can be high; and there are no formal procedures for conflict solving. When problems do arise, if staff are not flexible enough to fix them on their own then the manager has to fix the roster. 
Majority of methods for personnel scheduling belong to one of the following categories:

- Mathematical programming include Linear Programming, Integer Programming, Mixed Integer Programming, Column Generation, Branch-and-price, Dynamic Programming, Lagrange Relaxation, Goal programming. Integer programming is one of the most explored ones (e.g. Bard et al. 2003).

- (Meta-)heuristics include both constructive heuristics, and improvement heuristics such as Simulated Annealing, Tabu Search, Genetic Algorithm, and others. According to (Van den Bergh et al. 2013), Tabu search and Genetic Algorithms are methods preferred to Simulated Annealing.

- Artificial Intelligence include Constraint Programming (e.g. Laporte and Pesant 2004), Case-based Reasoning (e.g. Beddoe et al. 2009), etc.

- Other simulation is often combined with Queuing models to determine the staffing levels (e.g. Qi and Bard 2006).

- Hybridisation of methods if designed properly, a hybridisation approach takes advantage of the best of the used methods (e.g. Valouxis and Housos 2000).

One can notice that the research into personnel scheduling approaches is very prolific, resulting in a variety of methods which handle constraints and objectives in different ways. The question remains whether the models that have been developed so far represent the personnel scheduling problem in a realistic way and whether they capture all the issues faced by the managers/schedulers when assigning personnel to shifts.

\section{Software packages for personnel scheduling}

In the very recent past, in many institutions, rosters were constructed manually and were paper-based. In constructing rosters, schedulers employed undocumented rules, which put their rosters beyond scrutiny. However, the software market has recognised the need for software packages to assist in personnel scheduling. The existing commercially available software for rostering (or workforce optimisation in general) can be classified into three main classes: software for roster administration, software for workforce management, including workforce planning and design of shift patterns, and software for automated rostering. The purpose of each class of software will be described together with their common and/or individual salient characteristics.

\subsection{Software for roster administration}

Purpose Software from this class provides key tools to facilitate the preparation of rosters to meet a variety of staff and organisation requirements. It offers a wide range of roster presentation/reporting/monitoring features that are very useful for managers. However, such software cannot be used for automated rostering on its own. For each software it has to be investigated whether it can be linked with an external piece of software such as algorithm(s) for automated rostering.

Scheduling features Software in this class supports a variety of scheduling features, excellent reporting and interface with other relevant applications. Generally, scheduling features include accommodation of requests, preferences and flexible working patterns of staff. For example, it offers an interface for setting of working patterns, which can then be cascaded forward and varied as needed. Self-rostering is another scheduling feature often present in this class of software. All non-shift activities such as training can be also rostered and monitored. 
Annualised work hours or term-time working can also be accommodated and the software can track staff hours.

Reporting In general, software in this class offers a user colour-coded interface with different colours for skill groups, teams, etc, with indications of shifts which are below or above the target. It allows tracking how much time is being spent on non-shift activities and where. Also, it is important to track the hours of staff on annual work hours. An interesting feature in reporting is an audit trail which records details of changes to a published roster; for example who made what change and when. It also enables recording of any issues which arose during the previous shift and makes it available to the incoming shift manager.

Links with other software packages Generally, software provides link with the payroll system and can generate input to it containing accurate reports on sickness, training and other absence of staff.

\subsection{Software for workforce management}

Purpose Software for workforce management enables workforce planning and design of shift patterns. Generally, it provides configurable tools for workforce management. The software may also allow the user to define their own shift patterns with input data about resources (for example, the number of teams) and constraints from a given list related to the number of consecutive working shifts, days off, etc. The software can then generate a roster with given shift patterns and enables the evaluation and comparison of different rosters. Sometimes, such software can automatically generate rosters for less complex problems, which have limited set of constraints/rules to comply with, but its main purpose is shift planning and roster administration.

Reporting Software for workforce management show graphically the required staff levels and compare them with the rostered staff with a clear indication of under-staff and over-staff periods.

Features Additional tools that software from this class often contain are relevant to the capacity planning, leave management, time and attendance, and employee self-service modules. This software has a proven track-record in aviation (for ground workforce planning and scheduling) and ports. It serves as a very convenient tool for staff planning in the sense that it can, for example, determine the level of required airport resources based on flight schedules and passenger loads. It graphically shows the required coverage and rostered staff clearly indicating over-staff periods. It also offers monitoring features for staff re-rostering. However, although this software is useful for staff planning in aviation its capability for automated rostering has to be investigated. Some providers of workforce management software claim that their systems utilise the state-of-the-art optimisation techniques to generate the most cost effective schedules that balance staff availability to workload requirement and take into account employee contracts, availability and preferences, multi-skilling and staff competencies, working time regulations and union agreements, and rolling horizon of a given number of weeks $(4,8,13,17,26$ weeks etc.). However, they do not give any detail about rostering and what metrics are used in measuring the quality of rosters.

There is also software which assists in solving a wide range of workforce planning problems. It enables what-if analysis of all kinds of scenarios based on the demand for staff. It can generate multiple shift patterns by altering key variables including shift length, team sizes, minimum and maximum consecutive shifts or days off and preferred shift rotation. It provides a very convenient environment for running different scenario planning sessions. An example of a successful use of such a software in an airport was the reduction of 14 different initial rosters to 7 of them. The general feedback on the software was very positive in the sense that 
the new system has created a better work-home balance for employees, while giving them the reassurance of a set income. However, there is no evidence that explicit metrics for staff well-being were used.

\subsection{Software for automated rostering}

Purpose Software for automated rostering provides full support on assigning personnel to shifts to the scheduler. Desk research into the software market has revealed that there are only a limited number of software systems which provide fully automated rostering. Some software is general and flexible to a reasonably high extent and potentially could be useful for a wide range of organisations.

Scheduling features We list some of the features that a "good" piece of software from this class usually has. It automatically assigns staff to user-defined shift types complying with the rules introduced by the employer such as a required number of working hours per week, breaks, etc., and respecting preferences expressed by staff as much as possible. The software can also decide on the start and end times of shifts for each employee for each day. The demand/cover constraints can be specified by each activity (and optionally skill) for each user-defined time interval within the planning horizon (for example, every $15 \mathrm{~min}$ ). The software also provides break management, for example it can position breaks and assign activities within shifts according to the user-defined rules and preferences. It enables the user to define a wide range of scheduling constraints and preferences including different grades/skills, different contracts, different shift patterns, preferences, requests, etc. The constraints, preference and objective function are often defined by using XML format. The user can define the relative importance (weights) of components in the objective function. Objective function is usually linear or quadratic. Parts of the roster can be "frozen" and not changed while the method searches for an optimal roster. It may allow modelling of the constraint that two employees must have the same or different shifts. It allows rotation of a roster. It can model constraints at boundaries between planning periods, which is important for staff for whom the roster in the current planning period is affected by their roster in the previous planning period. This could be also used to monitor burn rate for annual work hours. It also supports changes to the initially generated roster; for example, it can suggest changes to repair late notice absence.

Reporting A good user interface highlights any violations of rules, and constraints. It also enables what-if analysis with different scenarios to investigate the impact of swapping or changing shift allocations.

Although majority of today's rostering systems have limited functionalities and also can be seen as a means for disempowerment and demotivation of staff, they can provide, if applied appropriately, safer, more robust rosters in an efficient manner and can enable staff to retain the control over when and how they work. Some of these systems can automatically generate rosters, but anecdotal evidence suggests that the software fills in on average only $60-65 \%$ of rosters (i.e. required shifts), so that the given rules/constraints are not violated. Sometimes $80-90 \%$ of the roster can be automatically generated, but even then the remaining part of the software still has to be filled in manually. An appropriate algorithm customised to address all the constraints in the given real-world personnel scheduling problem could create better rosters. 


\section{Towards bridging the gap between personnel scheduling research and practice}

The literature on personnel scheduling reveals that there are different ways in which researchers engage with practice. Some researchers collaborate with companies/scheduling managers, and a number of them report that their methods have been used in practice. Other researchers use real-world data available in the literature or Web sites, while the remaining researchers are focused on theoretical results.

In the published literature on personnel scheduling, researchers often present methods which are developed to deal with real-world data on personnel scheduling. Data are collected either from a single company or a number of companies. Similarly, in nurse rostering, methods have been developed for a single ward in a hospital, or one hospital, but some of them are developed for a number of hospitals. Kellogg and Walczak (2007) considered 50 methods for nurse rostering and reported that 7 out of the 15 implemented methods were in use in only one hospital, 4 were used in many hospitals, 2 were used in a single ward or a nursing unit, while 2 of them led to the development of commercial packages.

The timetabling community offers a couple of collections of benchmark instances which serve to the community to compare their algorithms with other state-of-the-art algorithms. Often in use are nurse rostering instances based on real-world data from hospitals which can be downloaded from the Web site http://www.cs.nott.ac.uk/ tec/NRP/index.html). Another Web page offers a generator for nurse rostering problems requiring the user to enter data about the size of problem, preferences and coverage (http://www.projectmanagement.ugent.be/nsp. php). This enables researchers to compare different approaches developed for nurse rostering. Two nurse rostering competitions organised in 2010 and 2014-15, also serve very well as sources of data (Web pages http://www.kuleuven-kulak.be/nrpcompetition and http://mobiz. vives.be/inrc2/). Randomly generated instances for the rotating personnel scheduling problems are presented on the Web site https://www.dbai.tuwien.ac.at/staff/musliu/benchmarks. In the published literature that report the results obtained on these instances, no method outperforms all the others on all instances. If the proposed method has the best performance on some of the instances it usually gives a green light for the method to be published in a scientific journal. This demonstrates advances in the timetabling community. However, it requires a lot of effort to implement the results of research in practice.

The literature on personnel scheduling reveals that a reasonable number of academic researchers develop their methods in collaboration with a company. However, it appears that only a small number of the published research into personnel scheduling have been implemented in practice. In their survey paper, Van den Bergh et al. (2013) stated that among 196 papers which were developed considering real-world based data, 46 of them were applied in practice, while 76 paper used theoretical data. This distribution is illustrated in a chart presented in Fig. 1.

A good example of a system which was developed in co-operation between a company and an institute and applied in practice is described in (Eveborn and Ronnqvist 2004). The system used branch-and-price algorithm with column generation and handled preferences of employees and fairness in scheduling. A particular feature of this system was that it allowed employees to change from one task to another during the assigned shift. This is a feature that is rarely considered in scheduling methods. The authors reported their experience in the implementation of the system in three companies: a zoo where employees were scheduled to work in shops, restaurants and ticket offices, a mail order company and a centre for telephone 


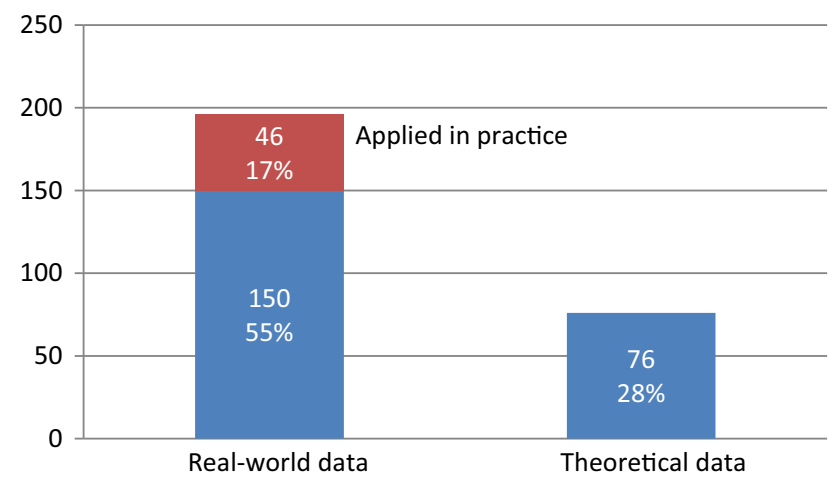

Fig. 1 Nature of data used for the development of methods for personnel scheduling (data taken from Van den Bergh et al. 2013)

banking. Their conclusion was that the speed of generating a solution was more important than the guarantee of optimality.

In their study, Kellogg and Walczak (2007) were focused on implementations of nurse rostering research in hospitals and concluded that there were more examples of successful knowledge transfer in Europe than in the United States. They supposed that the reason for this was that in Europe there were more hospitals that were members of universities than in the Unites States and that this facilitated implementation. In the United States, there are many profit oriented healthcare institutions which are not ready to experiment with unknown and untried methodologies.

One of the first optimisation-based air-crew rostering system was used by Air New Zealand and reported by Butchers et al. (2001). The authors state that they worked closely with all groups in the airline company affected by the introduction of the crew scheduling system: the scheduling staff, managers and crews. Even more they engaged with the representatives of the flight-attendant union who defined the roster quality measures to be used in the optimisation. They develop customised systems to solve all aspects of the planning and rostering problems for national and international flights and different type of crew members (pilots and flight attendants). Among them the problem of largest size involved 1500 flight attendants, and the time required to construct a roster of this size was reduced from several days of two people to several hours by the optimisation system. One can notice that the collaboration started with the sponsorship of student projects by the airline company, but then when the company recognised the benefits of the optimisation methods (not only financial but other sorts of intangible benefits) the collaboration continued with the employment of permanent operational research professionals.

\subsection{Research directions}

There are a number of possible reasons why there is still a gap between research into personnel scheduling and practice. Here we provide insights into some issues that arise in real-world personnel scheduling, but have not given yet a due attention in the research community. A discussion with schedulers and software developers about which of them should be pursued and which corresponding functionalities should be offered in a software package would be very useful. 


\subsubsection{Well-being of personnel}

There has been an increasing volume of evidence, mostly from occupational medicine, on the impact of improving staff well-being on organisational outcomes (Donald et al. 2005). In addition, there were studies which investigated the effects of shift work on health and safety (Harrington 2001), the effects of overtime work on hazard rates (Dembe et al. 2005), the effects of different shift lengths (Ellis 2008), night shifts (Gander et al. 2007), rest days (Tucker et al. 1996), the effects of delaying shift start and end times (Rosa et al. 1996), provided comparison between forward-rotating and backward-rotating schedules (Klein Hesselink et al. 2010), between fixed shifts and rotating shifts (Martens et al. 1999), between different shift patterns (Baxter and Mosby 1988), etc. Also, there has been an increasing interest of institutions to consider well-being of their employees. Generally, personnel scheduling methods consider a variety of constraints, while some of them are related to well-being of employees; for example a maximum number of days on or patterns that should be avoided like early shift after night shift. However, these algorithms do not consider employees' wellbeing in any formal or objective way. Petrovic et al. (2016) suggested four measures to be used in personnel scheduling: Work-life balance following on Cunningham's research (1982), fatigue and risk indicators introduced by Health and Safety Executive (HSE) in the United Kingdom (Spencer et al. 2006), and compliance with the HSE guidelines proposed based on their research into the effects of shift-work on employees (HSE 2006). Work-life balance measures the availability of quality free time for each employee and counts two day free weekends, free weekend days, free evenings, free blocks of at least two days and free days in the roster. The fatigue indicator is based on the Karolinska Sleepiness Scale (Akerstedt and Gillberg 1990) and relates to the probability of high levels of sleepiness. It is calculated by considering the start time of the shift, its length and the time of the day, the cumulative factor that considers the pattern of work which precedes each shift, and type of the job and breaks. The risk indicator, which is related to the occupational injury, is expressed in terms of the relative risk of an accident/incident occurring. HSE guidelines recommend good practice for shift work scheduling. They include no permanent night shifts, forward-rotating shifts instead of backward-rotating, maximum $12 \mathrm{~h}$ duration of shifts, and $8 \mathrm{~h}$ of night shifts where work is demanding, monotonous, dangerous, safety critical, no split shifts, etc. The experiments on real-world nurse rostering problems demonstrated that it was possible to generate rosters of high performance which also achieved good well-being measures for all personnel. However, in some problem instances the trade-off between the well-being measures and performance had to be made. The explicit consideration of well-being measures in the objective function enabled a better control of employees' well-being measures in the roster by assigning different weights to measures. An interesting observation was that an appropriate distribution of working shifts was very important for the employees' well-being leading to their improvement even with the increase of the number of working shifts of some employees.

\subsubsection{Integration of planning and scheduling}

Generally, service systems are labour intensive and demand for services has to be met when requested. Workforce planning/staffing deals with strategic decisions related to the optimal size of personnel and mix of their skills required to meet the forecasted demand for service and provide high quality service. In some sectors, especially the ones which are expected to provide service 24/7, shifts have also to be designed in such a way as to meet the demand (see for example Jagtenberg and Post 2016). The demand for service is usually expressed 
by statistical data. The required staffing levels can be expressed as a fixed number of staff, or minimal and maximum preferred number. One of the main differences between scheduling in manufacturing and scheduling in services is the way they are designed to meet the demand (Heizer and Render 2011). Manufacturing systems can use inventories to respond to fluctuations in demand. Opposite to them, many service systems do not have inventories. In some sectors, for example in nursing in healthcare, there are banks of nurses that can be utilised to meet the demand, but this a costly solution. The inability to store a service delivery as inventory means that the planned personnel, i.e. capacity, needs to meet closely the forecasted demand for service. Understaffing and overstaffing are undesired; the former leads to overworked staff and delays in service provision which result in lower quality of service, lower personnel's motivation for work, lower job satisfaction and consequently increase in personnel turnover; the latter causes excessive personnel cost. The planning precedes personnel scheduling, i.e. in scheduling it is assumed that staffing levels, personnel structure and shifts are given and they serve as input to scheduling. Relevant objectives and constraints such as personnel preferences, well-being of personnel, skills and knowledge required for a task, legal and organisational requirements, and consistency in service are then considered in scheduling. Consequently, scheduling is considerably affected by planning, and inappropriate planning leads to sub-optimal scheduling solutions.

The research into models which integrate planning and scheduling has started to attract the attention of the personnel scheduling research community, but it is still rather scarce. Castillo et al. (2009) developed an approach for personnel scheduling in a call centre. Traditionally, the aim is to minimise the labour cost subject to an acceptable service level, which is defined as the percentage of customers served within a predetermined time interval. In this research, the aim was to minimise the labour cost and maximise the service level simultaneously, together with other complementary objectives. This enabled the analysis of the interaction between cost and service quality. Their approach considered the demand profiles and use them to generate plausible schedules with various combinations of possible shifts to meet staff requirements. Beliën et al. (2012) applied a Mixed Integer Linear Programming - based enumeration approach to personnel scheduling for airline maintenance in which they introduced both personnel size and scheduling related decision variables. However, the problem they faced had some specific characteristics; namely, each maintenance activity had to occur within a given time window (this is a special coverage constraint), and the employees could be transferred to another department, which meant that the staffing decision could be made an integral part of the scheduling decision for a given planning horizon. The model developed was useful for the negotiation with the labour union about generally accepted schedules, but it was not clear if the company used it for generating schedules. Komarudin et al. (2013) developed an integrated methodology which evaluated the suitability of personnel structure determined in the planning phase while constructing schedules (rosters). The sources of violation of coverage requirements were examined to provide a feedback to the planning phase to change the personnel structure. The methodology was evaluated on two nurse rostering problems.

The generalisation of the methodology presented in (Komarudin et al. 2013) would mean that a metric to be used on the generated schedules have to be defined, which would provide a feedback to the planning phase and possibly suggest a change to the staffing level and/or personnel structure that would improve schedules. For example, one metric could measure the unmatched demand for a particular type of service in rosters over a period of time, and consequently recommend an increase/decrease of staff with specific characteristics (for example, particular personnel skills or particular shift type) to better address scheduling 


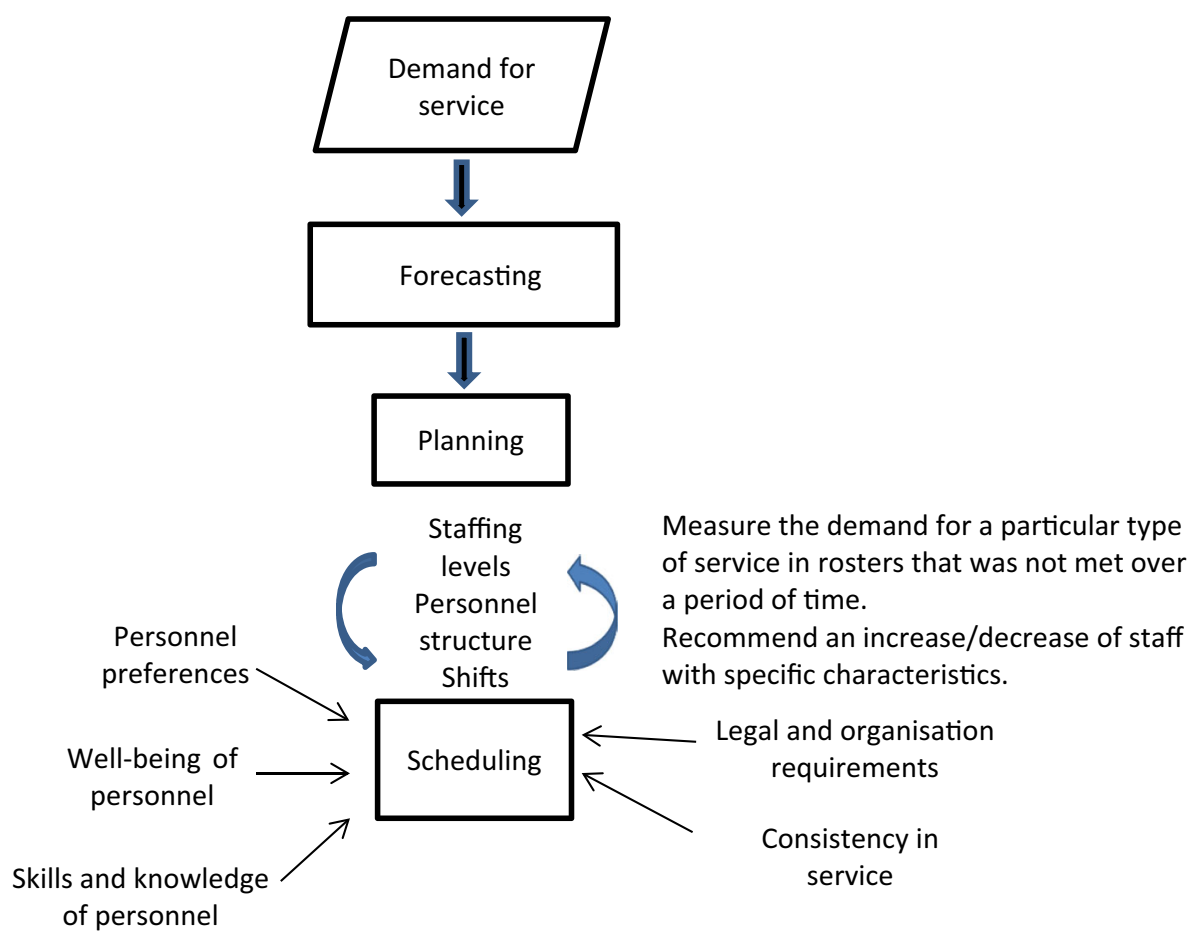

Fig. 2 A framework for integration of personnel planning and scheduling

constraints. A flowchart showing a framework for integration of personnel planning and scheduling is shown in Fig. 2.

\subsubsection{Integration of personnel scheduling with other related problems}

In many sectors, there is a need for systems that provide solutions to integrated problems. For example, the scheduling of surgeons and operating rooms should consider the availability of beds in the wards required for post-operation recovery (Belien and Demeulemeester 2007), and this scheduling should be considered together with nurse rostering because it heavily affects the workload of nurses (Carter 2002; Belien and Demeulemeester 2007). Scheduling of the personnel on shop floor should be integrated with machine scheduling (Artigues et al. 2007). An approach to modelling of vehicle routing together with personnel scheduling is described in (Eveborn et al. 2006, 2009). The research resulted in excellent commercial software for daily planning of health and social care at home. The authors reported an impressive increase in the efficiency and reduction of the cost in the institutions which have been using the developed software.

\subsubsection{Quality of a method/system for personnel scheduling}

The academic community focuses on the development of new methods for personnel rostering often aiming to produce effective solutions which outperform other methods on the benchmark instances. However, there are other criteria which should be used in the comparison of methods. Petrovic and Vanden Berghe (2012) suggested seven criteria: 
(1) Expressive power describes the ability of the model to represent a wide variety of constraints that are present in real-world personnel scheduling. Often methods are developed to handle simplified problems that do not reflect well the real-world. Vanden Berghe (2012) identified main challenges in automating the rostering process, which include the precise capturing of the actual required skills and experience in each time period of the roster, the correct modelling of complex hard and soft constraints to correspond to real-world environment (some of these constraints/requirements/expectations are freely formulated by the scheduler and it is difficult to formalise them in a model), and the capturing of the importance of objectives to correspond to the personnel perception of the roster quality. Therefore, models which do not capture adequately constraints and objectives present in real-world rostering or ignore some of them, are seen as one of the obstacles for the use of automated rostering systems in practice.

(2) Flexibility refers to the ability of the model/system to be applied to different personnel scheduling problems or to address the changes in the problem, which implies that the addition or removal of constraints should be easy. It also addresses the scalability issues of methods.

(3) Algorithmic power includes both effectiveness, i.e. the value of the objective function and efficiency, i.e. resources, usually computational time, required to generate a roster.

(4) Learning capabilities i.e. the capability of the system to learn and improve its performance over time.

(5) Maintenance refers to the ability of the system to update the knowledge of the problem and also to handle redundant and conflicting knowledge.

(6) Re-scheduling capability refers to the capability to react to the unforeseen disturbances in the environment (please see 5.1.3). Often in practice, the manager handles these cases manually which may have a negative impact on the performance of the roster.

(7) Parameter tuning the parameters usually have to be carefully tuned so that the method performs well over a wide range of problem instances. The sensitivity of the parameter setting may jeopardise the adoption of the method in practice.

\subsubsection{What-if analysis}

It would be very helpful to the decision maker/manager to be able to decide how to meet the demand in a given personnel scheduling problem and what a cost-effective option would be: overtime shifts, night shifts, or subcontracting. These can serve as parameters in a personnel scheduling method which is used as a simulation tool to generate results for different what-if scenarios. Visualisation can play an important role in presenting advantages and disadvantages of different options to managers.

\subsubsection{Re-scheduling}

Re-scheduling (also referred to as re-rostering in the personnel scheduling literature) is concerned with the maintenance of the roster after its approval (Clark and Walker 2011). Re-scheduling is needed to respond to occurrences that disturb the schedule such as unavailability of employee, higher demand for service than expected, etc. Post-approval changes can affect as many as $50 \%$ of the rostered shifts (Kerr and Timony 2009). Re-scheduling can involve the same range of activities as scheduling itself. The managers have to be informed about re-scheduling. There are a large number of possible solutions to choose from, but typically re-scheduling is done under stronger time pressure (de Snoo et al. 2011). Limited examples of research in re-scheduling include (Moz and Pato 2004, 2007). There are a number of issues to be considered in re-scheduling, which are rarely examined. For example, 
which changes can be made to the roster? How they affect the fairness and well-being of employees? Which additional performance measures are appropriate to use to evaluate the quality of new rosters? In production scheduling, stability metrics are defined to measure the difference between the old and the new schedule obtained as a result if re-scheduling. Can we benefit from these research results in measuring the performance of a new roster?

\subsubsection{Modelling of particular features of real-world personnel scheduling}

Generally, papers on personnel scheduling consider many constraints. However, not many of them incorporate breaks. Full time personnel need breaks, while part-timers usually need a meal break if their shift length exceeds a certain threshold. Thompson and Pullman (2007) investigated if scheduling breaks in advance offered advantages over scheduling them in real-time. Typically when scheduling breaks in real-time, if demand is lower than expected the surplus staff are allowed to take their break or go home early. The staff are kept longer or their breaks are postponed if demand is higher than expected. It was shown that consequences of not scheduling breaks in advance could be a less profitable deployment of personnel and assigning them in real-time might mean that they were not assigned at all. This might result in dissatisfied workforce or violations of contractual obligations, or if breaks were longer than needed it might cause less productive workforce. Scheduling breaks in advance increases considerably the complexity of the scheduling problem. However, the conclusion was that it was more advantageous to schedule them in advance. Their findings were supported by an anecdotal evidence drawn from commercial labour scheduling software.

Most of the concepts and methods developed for a full-time employee are not applicable to the working environments with part-time employees since they do not guarantee continued availability. Their contractual rules are not as strict as for permanent employees either. It is still important for the institution to accommodate the preferences of part-time employees because they will be more inclined to stay and work for an institution if it provides rosters that are tailored to their individual needs and preferences. There is rather limited literature on scheduling of part-time workers (Mohan 2008; Hojati and Patil 2011; Akbari et al. 2013). These problems led to new objective functions and constraints related to the limited availability of part-time employees, their less strict contracts, aim to closely meet the demand (i.e. to minimise overstaffing), a possibility of assigning to part-time employees more than one shift per day, in which case their productivity could be taken into consideration especially if they were assigned consecutive shifts in a day, etc. Considering the rapid growth of service industry, which in many sectors depends on part-time workers, it is to be expected that this type of personnel scheduling problems will gain in importance.

Allocation of shifts which aims to accommodate different and possibly conflicting personal preferences can be a sensitive issue. Therefore, fairness can be perceived as an important indicator of the quality of a roster. Drake (2014) analysed roster policies at 28 NHS trusts and reported that fairness was stated to be the most common policy objective. However, this was considered to be one of the major shortcomings in the existing approaches to rostering. There has been some research into fairness in personnel scheduling (e.g. Komarudin et al. 2014). In order to address this issue properly, the first question is how to define fairness, especially in the presence of employees' preferences for working/not working certain shifts when everyone's roster will have different characteristics. Usually, in the literature on personnel scheduling some simple measures have been in use such as the number of approved requests per person or rule breakages given as a percentage of shifts worked. One may notice that this offers a shallow perspective of fairness. Impersonal fairness implies equality of personnel in a roster. However, a typical question that arises health care institutions is if junior and senior nurses 
in a ward should be assigned to the same number of unsocial shifts? In addition, fairness is differently perceived in different institutions or even in different parts of the same institution. The literature on resource allocation (also referred to as "cake-cutting problem") introduces a series of measures of social fairness, some of which could be useful in measuring the fairness in rosters. For example, an allocation is envy-free if every involved player prefers its allocation to any other player's allocation; an allocation is equitable if all players have the same utility for their respective shares, or the measure can be based on unfairness feelings of the players who compare their allocation to other players' allocations by using their own utility functions (Hoang et al. 2016). This research avenue is still expected to offer more research results.

\subsubsection{Modelling of uncertainty}

Many real-word personnel scheduling problems face different sources of uncertainty (Van den Bergh et al. 2013). There can be uncertainty in demand which leads to the unpredictable workload (Yan et al. 2008; Ceschia and Schaerf 2012; Campbell 2011). For example, there is usually uncertainty in the duration of a machine repair activity of the engineer, uncertainty in the length of calls in a call centre, uncertainty in the duration of a visit in domiciliary care, uncertainty in the length of stay of a patient in a ward, etc. Opposite to the manufacturing environment where inventories can help smoothing the demand, many service systems do not have inventories and cannot have more staff than available. One way to address this problem is to have multi-skills trained personnel who can undertake a number of tasks. Wallace and Whitt (2005) provided an analysis of the coordination of skill set design, staffing, and routing decisions in multi-skill call centres. Uncertainty in the demand is usually considered together with uncertainty in arrivals. For example, there is uncertainty in occurrence of failures on the machines, in arrival of calls, in patient arrivals, etc. There is also uncertainty of capacity, i.e. there is often the difference between planned and actual available staff. However, this source of uncertainty has been addressed only to a limited extend in the published literature on personnel scheduling.

\subsubsection{Maintenance of the software}

In order to be applied, there is a need to integrate the developed method with the existing licenced software in the institution. It usually means that the software vendor has to be contacted to enable the communication between different pieces of the software.

As is the case with all software packages, there is a need for a number of services to be provided such as training of the end-user (customer) to use the system, regular customer support, maintenance and the upgrade, i.e. extension of the features of the software, etc. This is something that generally academics are not placed well to provide. Partnership with software companies looks as a viable option to enter the personnel scheduling software market.

\section{Concluding remarks}

In many institutions, personnel scheduling is time consuming job that often takes time from the primary role of the manager/scheduler. A typical example are head nurses who generally prefer to spend time taking care of patients instead of generating rosters. The use of computerised systems for personnel scheduling has been increasing, but they are still underutilised and often require considerable inputs from schedulers. Currently, the majority of 
the software for personnel scheduling offers more administrative assistance than automation of the roster generation. The question remains how do we make the best use of fruits of our research? In order to bridge the gap that still exists between research into personnel scheduling and practice, the community needs to engage more with managers/schedulers in practice to investigate all the complex issues that appear in real-world personnel scheduling and to consider them in the models and methods. Perhaps, the usefulness of the automated personnel scheduling systems would be better appreciated if the business key performance indicators relevant to scheduling are defined. One promising way forward is also to establish links with software developers who would assist in the implementation of developed methods for personnel scheduling.

Open Access This article is distributed under the terms of the Creative Commons Attribution 4.0 International License (http://creativecommons.org/licenses/by/4.0/), which permits unrestricted use, distribution, and reproduction in any medium, provided you give appropriate credit to the original author(s) and the source, provide a link to the Creative Commons license, and indicate if changes were made.

\section{References}

Akbari, M., Zandieh, M., \& Dorri, B. (2013). Scheduling part-time and mixed-skilled workers to maximize employee satisfaction. The International Journal of Advanced Manufacturing Technology, 64(5), 10171027.

Akerstedt, T., \& Gillberg, M. (1990). Subjective and objective sleepiness in the active individual. International Journal of Neuroscience, 52, 29-37.

Artigues, C., Gendreau, M., \& Rousseau, L-M., (2007). A flexible model and a hybrid exact method for integrated employee timetabling and production scheduling. In: E.K. Burke and H. Rudová(Eds.): PATAT 2006, LNCS Springer 3867, 67-84.

Bailyn, L. (2007). Self-scheduling system for hospital nurses: An attempt and its difficulties. Journal of Nursing Management, 15, 72-77.

Bard, J., Binici, C., \& de Silva, A. (2003). Staff Scheduling at the United States Postal Service. Computers \& Operations Research, 30(5), 745-771.

Baxter, J., \& Mosby, M. (1988). Generating acceptable shift-working schedules. Journal of the Operational Research Society, 39, 537-542.

Beddoe, G., Petrovic, S., \& Li, J. (2009). A hybrid metaheuristic case-based reasoning system for nurse rostering. Journal of Scheduling, 12(2), 99-119.

Beliën, J., Cardoen, B., \& Demeulemeester, E. (2012). Improving workforce scheduling of aircraft line maintenance at sabena technics. Interfaces, 42(4), 352-364.

Belien, J., \& Demeulemeester, E. (2007). Building cyclic master surgery schedules with leveled resulting bed occupancy. European Journal of Operational Research, 176(2), 1185-204.

Belien, J., \& Demeulemeester, E. (2008). A branch-and-price approach for integrating nurse and surgery scheduling. European Journal of Operational Research, 189(2008), 652-668.

Burke, E., De Causmaecker, P., Vanden Berghe, G., \& Van Landeghem, H. (2004). The state of the art of nurse rostering. Journal of Scheduling, 7(6), 441-499.

Butchers, E. R., Day, P. R., Goldie, A. P., Miller, S., Meyer, J. A., Ryan, D. M., et al. (2001). Optimized crew scheduling at air New Zealand. Interface, 31, 30-56.

Campbell, G. M. (2011). A two-stage stochastic program for scheduling and allocating cross-trained workers. Journal of the Operational Research Society, 62, 1038-1047.

Carter, M. (2002). Health care: Mismanagement of resources. ORMS Today, 19, 26-32.

Castillo, I., Joro, T., \& Li, Y. Y. (2009). Workforce scheduling with multiple objectives. European Journal of Operational Research, 196, 162-170.

Ceschia, S., \& Schaerf, A. (2012). Modeling and solving the dynamic patient admission scheduling problem under uncertainty. Artificial Intelligence in Medicine, 56(3), 199-205.

Clark, A., \& Walker, H. (2011). Nurse rescheduling with shift preferences and minimal disruption. Journal of Applied Operational Research, 3(3), 148-162.

Cunningham, J. B. (1982). Compressed shift schedules: Altering the relationship between work and non-work. Public Administration Review, Wiley, 42(5), 438-447. 
de Snoo, C., van Wezel, W., Wortmann, J. C., \& Gaalman, G. J. C. (2011). Coordination activities of human planners during rescheduling: Case analysis and event handling procedure. International Journal of Production Research, 49(7), 2101-2122.

Dembe, A. E., Erickson, J. B., Delbos, R. G., \& Banks, S. M. (2005). The impact of overtime and long work hours on occupational injuries and illnesses: new evidence from the United States. Occupational Environmental Medicine, 6, 588-597.

Dolan, P., Layard, R., \& Metcalfe, R. (2011). Measuring subjective well-being for public policy. Office for National Statistics, 1-19.

Donald, I., Taylor, P., Johnson, S., Cooper, C., Cartwright, S., \& Robertson, S. (2005). Work environments, stress, and productivity: An examination using ASSET. International Journal of Stress Management, 12(4), 409-423.

Drake, R. (2014). Five dilemmas associated with e-rostering. Nursing Times, 110(20), 14-16.

Edie, L. C. (1954). Traffic delays at toll booths. Operations Research, 2, 107-138.

Ellis, J. R. (2008). Quality of care, nurses' work schedules, and fatigue: A white paper, seattle: Washington state nurses association, 1-24.

Ernst, A. T., Jiang, H., Krishnamoorthy, M., Owens, B., \& Sier, D. (2004a). An annotated bibliography of personnel scheduling and rostering. Annals of Operations Research, 127, 21-144.

Ernst, A. T., Jiang, H., Krishnamoorthy, M., \& Sier, D. (2004b). Staff scheduling and rostering: A review of applications, methods and models. European Journal of Operational Research, 153(1), 3-27.

Eveborn, P., Flisberg, P., \& Ronnqvist, M. (2006). LAPS CARE-an operational system for staff planning of home care. European Journal of Operational Research, 171, 962-976.

Eveborn, P., \& Ronnqvist, M. (2004). Scheduler-A system for staff planning. Annals of Operations Research, $128,21-45$.

Eveborn, P., Ronnqvist, M., Einarsdottir, H., Eklund, M., Liden, K., \& Almroth, M. (2009). Operations research improves quality and efficiency in home care. Interfaces, 39(1), 18-34.

Gander, P., Purnell, H., Garden, A., \& Woodward, A. (2007). Work patterns and fatigue-related risk among junior doctors. Occupational and Environmental Medicine., 64(11), 733-738.

Gopalakrishnan, B., \& Johnson, E. (2005). Airline crew scheduling: State-of-the-art. Annals of Operations Research, 140, 305-337.

Harrington, J. (2001). Health effects of shift work and extended hours of work. Occupational and Environmental Medicine, 58(1), 68-72.

Heizer J., \& Render, B. (2011). Operations Management, Pearson, 10th Edition.

Hoang, L. N., Soumis, F., \& Zaccour, G. (2016). Measuring unfairness feeling in allocation problems. Omega, 65, 138-147.

Hojati, M., \& Patil, A. S. (2011). An integer linear programming-based heuristic for scheduling heterogeneous, part-time service employees. European Journal of Operational Research, 209, 37-50.

HSE (Health and Safety Executive) (2006). Managing shiftwork, Health and safety guidance, service code HS6256, The Office of Public Sector Information, Information Policy Team, ISBN:9780717661978, (http://www.hse.gov.uk/pubns/books/hsg256.htm) accessed 7 Nov 2016.

Hung, R. (2002). A note on nurse self-scheduling. Nursing Economics, 20(1) 37-39.

Jagtenberg C., \& Post, G., (2016). Workforce planning from sales to schedules, In: (Eds.) E. Burke, L. Di Gaspero, E. Özcan, B. McCollum, A. Schaerf, Proceedings of the 11th international conference on practice and theory of automated timetabling (PATAT 2016), Udine, Italy, Aug 23-26, pp. 493-498.

Kellogg, D. L., \& Walczak, S. (2007). Nurse scheduling: From academia to implementation or not? Interfaces, 37(4), 355-369.

Kerr, F., \& Timony, Y. (2009). Review of an automated rostering system from a nurse manager's perspective. In K. Saranto, P. Flatley Brennan, H-A. Park, M. Tallberg, \& A. Ensio (Eds.), Connecting Health and Humans, Vol. 146, Series studies in health technology and informatics, IOS Press Ebooks, 96-102.

Klein Hesselink, J., de Leede, J., \& Goudswaard, A. (2010). Effects of the new fast forward rotating five-shift roster at a dutch steel company. Ergonomics, 53(6), 727-738.

Komarudin, Guerry, M.-A., De Feyter, T., \& Vanden Berghe, G. (2013). The roster quality staffing problem-A methodology for improving the roster quality by modifying the personnel structure. European Journal of Operational Research, 230, 551-562.

Komarudin, Guerry, M-A., Smet, P., De Feyter, T., \& Vanden Berghe, G. (2014). A two-phase heuristic and a lexicographic rule for improving fairness in personnel rostering. In E. Ozcan, E. Burke, B. McCollum, (Eds), Proceedings of the 10th international conference on the practice and theory of automated timetabling. Practice and Theory of Automated Timetabling (PATAT2014) York, United Kingdom, 26-29 Aug, 292-308.

Laporte, G., \& Pesant, G. (2004). A general multi-shift scheduling system. Journal of the Operational Research Society, 55, 1208-1217. 
Martens, M. F. J., Nijhuis, F. J. N., van Boxtel, M. P. J., \& Knottnerus, J. A. (1999). Flexible work schedules and mental and physical health. A study of a working population with non-traditional working hours. Journal of Organizational Behavior, 20(1), 35-46.

Mohan, S. (2008). Scheduling part-time personnel with availability restrictions and preferences to maximize employee satisfaction. Mathematical and Computer Modelling., 48, 1806-1813.

Moz, M., \& Pato, M. V. (2004). Solving the problem of rerostering nurse schedules with hard constraints: new multicommodity flow models. Annals of Operations Research, 128, 179-197.

Moz, M., \& Pato, M. V. (2007). A genetic algorithm approach to a nurse rerostering problem. Computers \& Operations Research., 34, 667-691.

Musliu, N. (2006). Heuristic methods for automatic rotating workforce scheduling. International Journal of Computational Intelligence Research, 2(4), 309-326.

Musliu, N. (2013). Applying machine learning for solver selection in scheduling. In: proceedings of 10th metaheuristics international conference (MIC 2013), Singapore, 5-8 Aug, 2013.

Petrovic S., Parkin J. \& Curtois T. (2016). Enhancement of employee timetabling with well-being measures. In the procedings of the 5th world conference on production and operations management P\&OM, Havana, Cuba, Sep 6-10, 2016.

Petrovic, S., \& Vanden Berghe, G. (2012). A comparison of two approaches to nurse rostering problems. Annals of Operations Research, 194, 365-384.

Qi, X. T., \& Bard, J. F. (2006). Generating labor requirements and rosters for mail handlers using simulation and optimization. Computers \& Operations Research, 33, 2645-2666.

Rosa, R. R., Härmä, M., Pulli, K., Mulder, M., \& Näsman, O. (1996). Rescheduling a three shift system at a steel rolling mill: Effects of a one hour delay of shift starting times on sleep and alertness in younger and older workers. Occupational and Environmental Medicine, 53(10), 677-685.

Silvestro, R., \& Silvestro, C. (2000). An evaluation of nurse rostering practices in the national health service. Journal of Advanced Nursing, 32(3), 525-535.

Smet, P., Bilgin, B., De Causmaecker, P., \& Vanden Berghe, G. (2014). Modelling and evaluation issues in nurse rostering. Annals of Operations Research., 218(1), 303-326.

Spencer, M. B., Robertson, K. A., \& Folkard, S. (2006). The development of a fatigue/risk index for shiftworkers. Health and Safety Executive (HSE) Research Report, 446, 1-82.

Thompson, G. M., \& Pullman, M. E. (2007). Scheduling workforce relief breaks in advance versus in real-time. European Journal of Operational Research, 181, 139-155.

Tucker, P., Barton, J., \& Folkard, S. (1996). Comparison of eight and 12 hour shifts: Impacts on health, wellbeing, and alertness during the shift. Occupational and Environmental Medicine, 53(11), 767-772.

Utley M. (2016) Methods for modelling health service delivery: approaches, shortcomings and emerging opportunities, https://www.mrc.ac.uk/documents/pdf/utley-health-service-modelling-vignette/,paper commissioned for the Medical Research Council to provide an overview of areas where Operational Research modelling can help improve health services, Accessed 7 Nov 2016.

Valouxis, C., \& Housos, E. (2000). Hybrid optimization techniques for the workshift and rest assignment of nursing personnel. Artificial Intelligence in Medicine, 20(2), 155-175.

Van den Bergh, J., Belien, J., De Bruecker, P., Demeulemeester, E., \& De Boeck, L. (2013). Personnel scheduling: A literature review. European Journal of Operational Research, 226, 367-385.

Vanden Berghe, G. (2012). Personnel scheduling Challenging combinatorial optimisation problems with a personnel scheduling component, In (Eds.) D. Kjenstad, E. Burke, B. McCollum, Proceedings of the 9th International Conference on the Practice and Theory of Automated Timetabling (PATAT 2012), Son, Norway, 28-31 Aug 2012, pp. 10-14.

Wallace, R. B., \& Whitt, W. (2005). A staffing algorithm for call centers with skill-based routing. Manufacturing and Service Operations Management., 7(4), 276-294.

Yan, S. Y., Chen, C. H., \& Chen, M. J. (2008). Stochastic models for air cargo terminal manpower supply planning in long-term operations. Applied Stochastic Models in Business and Industry, 24, 261-275. 